\title{
Alternating hemiplegia of childhood
}

\section{Clinical case and video description}

Kelvin Au, MD; Tamara Pringsheim, MD, FRCPC

A

9-year-old girl was referred for evaluation of paroxysmal events of several types with the onset beginning in the first few days of life. She was the result of a normal pregnancy born at term with healthy nonconsanguineous parents. She had a history of global developmental delay and a few remote generalized tonic-clonic seizures. Her events evolved over time and increased in frequency and duration over the years. Between events, she returned to her baseline level of function. Her events were of various types, lasting seconds to minutes (video at Neurology.org/cp), including the following:

1. Arching of the back: These events were noted by her parents in the first few days of life.

2. Right body stiffening: The patient had her first event at age 17 months. From age 3, there was an associated head turn to the right, or left. Episodes lasted up to 10 minutes.

3. Right (or left)-sided weakness: These events began at age 8, and would start with right (or left) arm weakness, followed by facial weakness, and drooling. The patient often would also have speech arrest during her episodes, upon which she would type on a mobile device to communicate. Eventually her foot would become rigid. Arching of the back occurred with prolonged attacks. Ninety percent of events involved the right side. Left-sided events had similar phenomenology.

4. Vertical ocular oscillations: These began at 6 months of age. The patient's eyes would rhythmically oscillate vertically for 10-20 minutes.

The patient was awake, aware, and able to interact during events. Events were triggered by bright lights, fatigue, stress, and changes in temperature. Going to sleep would end an attack. She was seen by several neurologists and had failed trials of multiple antiepileptic drugs. Multiple MRIs and both awake and sleep-deprived EEGs were normal. Her general examination was unremarkable without dysmorphic features. Her neurologic examination revealed a mild mixed spastic and ataxic dysarthria and mild increased tone in her lower extremities bilaterally with hyperreflexia. Power was normal, but coordination was poor, with slow and imprecise movements. Gait was wide-based.

The patient's hemiplegic episodes, beginning at age 8 , were initially all right-sided. Her stiffening spells, which began as a toddler, had also been purely right-sided. It was only when the patient had a left-sided attack, witnessed by a physician, that alternating hemiplegia of childhood (AHC) was suspected and consent was obtained for sequencing of the ATP1A3 gene (implicated in AHC). The patient had a de novo c.2411C $>\mathrm{T}$, p.Thr804Ile heterozygous mutation in exon 17 of the ATP1A3 gene on chromosome 19. Mutations in the $\alpha 3$ subunit are associated with AHC. AHC is a rare disorder that is characterized by hemiplegia

\section{Practical Implications}

Paroxysmal ocular movements are an early suggestive symptom of alternating hemiplegia of childhood (AHC). AHC frequently coexists with epilepsy and it is important to caution against discounting novel spells as seizures in order to avoid a delay in diagnosis.

\section{Video}

Neurology.org/cp

Departments of Clinical Neurosciences (KA, TP) and Psychiatry, Pediatrics, and Community Health Sciences (TP), University of Calgary, Canada.

Funding information and disclosures are provided at the end of the article. Full disclosure form information provided by the authors is available with the full text of this article at Neurology.org/cp.

Correspondence to: tmprings@ucalgary.ca 
of either side of the body, paroxysmal tonic or dystonic spells, and oculomotor abnormalities with developmental delay. Onset occurs before 18 months of age. Evolution of paroxysmal neurologic symptoms and signs over time is typical for AHC. ${ }^{1}$

The differential diagnosis of AHC includes familial hemiplegic migraine (FHM) syndromes (FHM1-CACNA1A; FHM2-ATP1A2), episodic ataxia type 6, glutamate transporter disorders (SLC1A3), glucose transporter defects, GLUT1 deficiency (SLC2A1), infantile onset epileptic encephalopathies, severe myoclonic epilepsy of infancy (Dravet syndrome), SCN1A mutation, mitochondrial disorders, and disorders of dopamine biosynthesis. ${ }^{2}$

Treatment with flunarizine was helpful for this patient in decreasing the frequency of events. Diazepam was used as needed to help abort prolonged attacks. Other proposed treatments in AHC include anticonvulsants such as topiramate, though this has not been formally tested. ${ }^{1,2} \mathrm{~A}$ delay in diagnosis in patients with $\mathrm{AHC}$ is common due to frequent comorbid epilepsy and discounting of novel spells as seizures. Paroxysmal ocular movements are an early suggestive symptom of AHC. The long-term prognosis of AHC is generally poor due to recurrent attacks causing stepwise neurologic dysfunction and developmental delay. Medication may help reduce the frequency and severity of attacks, but whether it affects the long-term outcome of this disease is yet to be formally studied.

\section{REFERENCES}

1. Tenney JR, Schapiro MB. Child neurology: alternating hemiplegia of childhood. Neurology 2010;74: e57-e59.

2. Sweney MT, Newcomb TM, Swoboda KJ. The expanding spectrum of neurological phenotypes in children with ATP1A3 mutations, alternating hemiplegia of childhood, rapid-onset dystonia-parkinsonism, CAPOS and beyond. Pediatr Neurol 2015;52:56-64.

Received October 17, 2016. Accepted in final form January 11, 2017.

\section{AUTHOR CONTRIBUTIONS}

K. Au: Manuscript draft. T. Pringsheim: Project conception, organization, execution and manuscript review and critique.

\section{STUDY FUNDING}

No targeted funding reported.

\section{DISCLOSURES}

K. Au reports no disclosures. T. Pringsheim serves on the editorial boards of Neurology ${ }^{\circledR}$ Clinical Practice and Canadian Journal of Psychiatry and receives research support from Canadian Institutes of Health Research, Sick Kids Foundation, and Alberta Mental Health Strategic Clinical Network. Full disclosure form information provided by the authors is available with the full text of this article at Neurology.org/cp.

\section{Practical Implications}

Neurology ${ }^{\circledR}$ Clinical Practice is committed to providing clinical insights that are helpful to neurologists in everyday practice. Each Full Case will include a "Practical Implications" statement, a pearl of wisdom for the practicing clinician. 